\section{Revealing retroperitoneal liposarcoma morphology using optical coherence tomography}

\author{
Esteban F. Carbajal, ${ }^{a}$ Stepan A. Baranov, ${ }^{a}$ \\ Venu G. R. Manne, ${ }^{a}$ Eric D. Young, ${ }^{b}$ Alexander J. Lazar, ${ }^{b}$ \\ Dina C. Lev, ${ }^{\mathrm{b}}$ Raphael E. Pollock, ${ }^{\text {' }}$ and Kirill V. Larin ${ }^{\mathrm{a}, \mathrm{c}}$ \\ a University of Houston, Department of Biomedical Engineering, \\ N308 Engineering Building 1, Houston, Texas 77204 \\ ${ }^{b}$ The University of Texas M. D. Anderson Cancer Center, Sarcoma \\ Research Center, 1515 Holcombe Blvd, Houston, Texas 77030 \\ 'Saratov State University, Institute of Optics and Biophotonics, \\ Saratov 410012, Russia
}

\begin{abstract}
A new approach to distinguish normal fat, welldifferentiated (WD), and dedifferentiated liposarcoma (LS) tumors is demonstrated, based on the use of optical coherence tomography (OCT). OCT images show the same structures seen with conventional histological methods. Our visual grading analysis is supported by numerical analysis of observed structures for normal fat and WDLS samples. Further development could apply the real-time and high resolution advantages of OCT for use in liposarcoma diagnosis and clinical procedures. ( 2011 Society of Photo-Optical Instrumentation Engineers (SPIE). [DOI: 10.1117/1.3541789]
\end{abstract}

Keywords: liposarcoma; optical coherence tomography; retroperitoneal; cancer; detection.

Paper 10486LR received Sep. 2, 2010; revised manuscript received Nov. 18, 2010; accepted for publication Dec. 23, 2010; published online Feb. 16, 2011

Liposarcomas (LS) are a heterogeneous group of malignant mesenchymal neoplasms exhibiting features of adipocytic differentiation. ${ }^{1}$ In a current World Health Organization classification four major groups are defined: well-differentiated (WDLS), de-differentiated (DDLS), myxoid/round cell, and pleomorphic liposarcomas. ${ }^{2}$ The first two LS, WDLS and DDLS, usually develop in the abdomen/retroperitoneum (A/RP) and account for more than $90 \%$ of LS detected in such areas. ${ }^{3,4}$ Surgical resection is the most effective therapy for patients with abdominal/retroperitoneal LS, but the presence of contiguous organs, such as the kidney, pancreas, spleen, as well as the frequent recurrence of LS in A/RP suggests that enhanced surgical techniques are needed to minimize the extent of resection as well as decrease the incidence of LS recurrence. ${ }^{4,5}$ One of the key reasons underlying the high recurrence rate within the A/RP is the difficulty in delineating the margins of negative tumor resection intra-operatively due to the macroscopic resemblance of WDLS and DDLS to normal fat tissues. The tumor margin is commonly understood as the border between cancerous and normal tissues. Detection of margins is possible only by use of frozen section tissue biopsy which introduces significant delays for the completion of surgical resection and is also fraught with sampling inaccuracies, thereby introducing a measure of uncertainty as to the extent of surgical resection needed to achieve tumor control. ${ }^{5}$ Macroscopically, radiography, computed tomography, ultrasonography (US), Doppler US, positron emission tomography, and magnetic resonance imaging are used to pre-operatively detect and evaluate the LS in A/RP, but the resolution of these methods does not allow microscopic assessment of LS margins, suggesting the necessity of alternative methodologies such as optical coherence tomography (OCT). ${ }^{4}$ OCT is a microscopic, high-resolution, cross-sectional, real-time imaging modality allowing acquisition of 2-D and 3-D images of biological tissues up to several millimeters in depth at a micrometer scale. In the past several years, multiple OCT-based techniques have been proposed for classification of dense tissues, for detection and evaluation of different types of cancers. This suggests great potential for OCT to detect and evaluate human malignancy in situ. ${ }^{6}$

To the best of our knowledge there are no publications regarding liposarcoma tumors classification based on OCT modality. In this letter we demonstrate a new OCT-based approach that enables differentiating normal fat, WDLS, and DDLS in human tissues dissected from the A/RP area.

A spectral-domain OCT (SD-OCT) system (Fig. 1) was used in this study. A broadband superluminescent laser diode with a center wavelength at $\lambda_{c}=840 \mathrm{~nm}$ (Superlum, S840-B-I-20), a spectral bandwidth of $\Delta \lambda=50 \mathrm{~nm}$, and an output power of $20 \mathrm{~mW}$, was used in the OCT system which was based on a Michelson interferometer with $50 \%$ of the power going to the reference arm and $50 \%$ to the sample arm where the tissue was placed. Interference fringes formed after recombination of the backreflected and the backscattered light from the reference and the sample arm, respectively, were detected by a CCD camera based spectrometer and digitized using an analog-to-digital converter (NI-IMAQ PCI-1428). The spectrometer consisted of a diffracting grating (Wasatch Photonics, 1200 grooves per mm) and a CCD line scan camera (Basler L104K-2k, 2048 pixel resolution, $29.2 \mathrm{kHz}$ line rate). OCT A-scans (1D depth profiles) were obtained by computing the discrete Fourier transformation of the interferograms detected by the spectrometer, after converting the data to linear $k$-space using laser spectra information, and 2-D images (B-scans) and 3-D structures (C-scans) were constructed by lateral scanning which was performed by the galvanometer mirrors mounted in the sample arm over the area of up to $3 \mathrm{~mm} \times 3 \mathrm{~mm}$. The full imaging depth was about $3.4 \mathrm{~mm}$ in air and about $2.2 \mathrm{~mm}$ in tissue.

Tumor samples were taken after surgical resection at the University of Texas M. D. Anderson Cancer Center (UTMDACC) hospital and were kept in sterile phosphate buffered saline until they were imaged on the same day using the SDOCT system. Protocols for tissue processing were approved by the UTMDACC and University of Houston Biosafety Committees. After imaging, all tissue samples were formalin fixed and paraffin embedded, then prepared by routine methods for histological analysis. The region imaged using OCT was matched

Address all correspondence to: Kirill V. Larin, University of Houston, Department of Biomedical Engineering, N308 Engineering Building 1, Houston, TX 77204. E-mail: klarin@uh.edu

1083-3668/2011/16(2)/020502/3/\$25.00 @ 2011 SPIE 


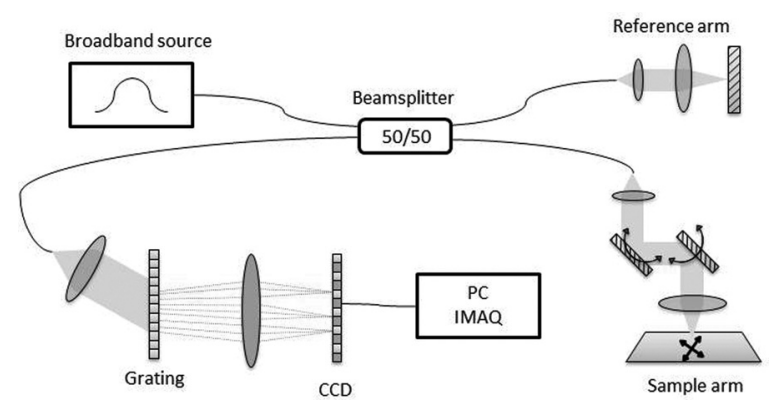

Fig. 1 Schematic of SD-OCT experimental system.

to the corresponding region on an H\&E-stained slide. Histologic diagnosis and classification of samples was performed by a UTMDACC sarcoma pathologist.

Representative OCT images of the resected human tissues are shown in Figs. 2, 3 and 4 with corresponding H\&E photomicrographs. One can note the similarity of obtained OCT and H\&E images, thus indicating the possibility of using OCT based modality as a replacement of histological analysis. From Fig. 2(a) of normal fat tissue and Fig. 3(a) of WDLS, the similar adipocytic cell patterns can be observed. Only slight differences such as the thickened fibrous septa and greater deviation in cell sizes for WDLS can be noted compared to normal fat. Such minor changes in characteristics of WDLS have been described previously, confirming the resemblance of normal fat and WDLS samples. ${ }^{7,8}$ Fluorescent in situ hybridization (FISH) analysis was performed using a MDM2 probe to confirm distinguishing between WDLS and normal fat. MDM2 gene amplification is present in both WDLS and DDLS, thus FISH is frequently incorporated as a component of the liposarcoma diagnostic assessment. ${ }^{9}$ DDLS samples (Fig. 4) depict highly cellular masses lacking adipocytic differentiation. The dense tissue and undefined structures are easily distinguished from WDLS and normal fat. However, the structural pattern of the DDLS is difficult to distinguish from connective tissues. Future studies will utilize these initial observations to help develop OCT intra-operative identification of DDLS as well as DDLSconnective tissue margins.

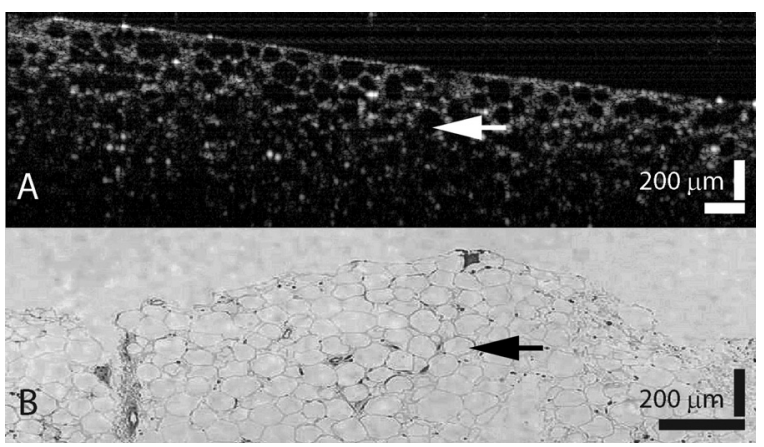

Fig. 2 (a) 2-D OCT structural image for normal fat. (b) H\&E histology section corresponding to (a). White arrow: adipocytic cell shape. Black arrow: adipocyte.

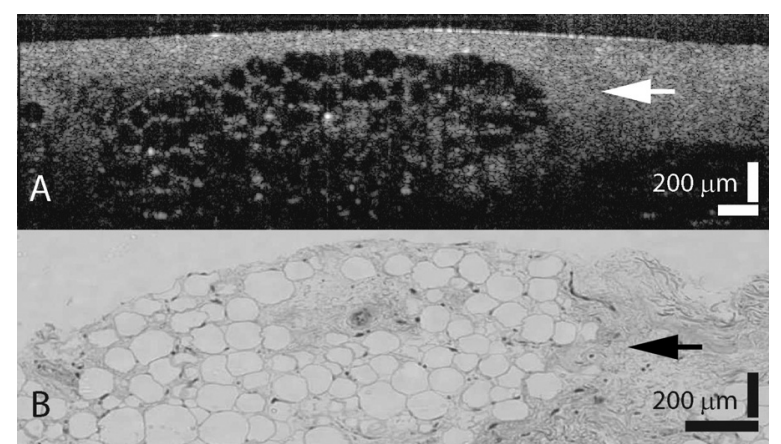

Fig. 3 (a) 2-D OCT structural image for WDLS. (b) H\&E histology section corresponding to (a). Black and white arrows: fibrous tissue.

A simple numerical method based on cell-counting was utilized to characterize WDLS and normal fat tissue samples. Five structural OCT B-scans for each sample were selected and the number of distinct adipocytic cell shapes in each B-scan were calculated using image-processing software. WDLS samples $(n=4)$ had an average number of $19.8 \pm 3.4$ adipocytic cell shapes $/ \mathrm{mm}^{2}$; normal fat samples $(n=4)$ had an average of $26.4 \pm 1.0$ adipocytic cell shapes $/ \mathrm{mm}^{2}$, demonstrating a significant difference between these two tissue types $(P<0.01$; Fig. 5). The decreased number of adipocytic cell shapes in WDLS can be explained by the main morphological features of WDLS, which has characteristically thickened septa, thereby reducing the aggregate area covered by adipocytic cells. Moreover, the adipocytic cells in WDLS are typically larger and more variable in size than normal fat cells, also accounting for the cell counting differences between WDLS and normal fat as observed with OCT assessment. ${ }^{7,8}$

In this study we manually selected the regions of WDLS and normal fat tissue samples for cell-counting used for proofof-principle purposes. By automation of cell counting analysis in the case of WDLS tumors, the OCT based techniques can be potentially utilized in clinical practice as in vivo and noninvasive microscopic imaging modality to identify the margins of WDLS tumors within affected normal fat tissues. Further studies will be focused on implementing an automatic cell counting algorithm and probe-integrated imaging methods, which would help in detecting early stages of liposarcoma.

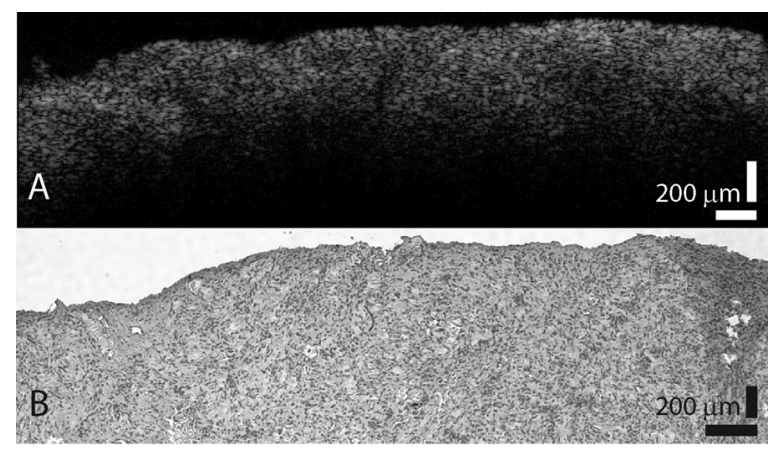

Fig. 4 (a) 2-D OCT structural image for DDLS. (b) H\&E histology section corresponding to (a). 


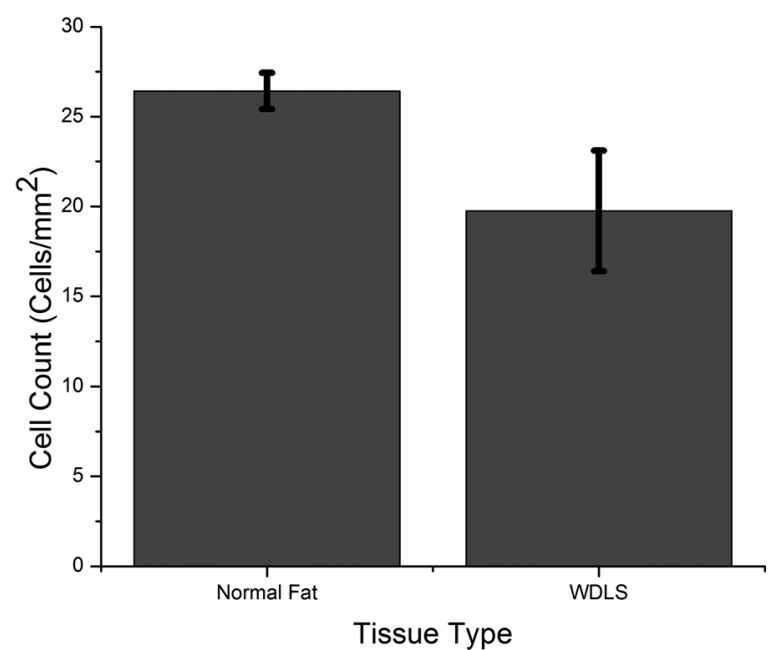

Fig. 5 Cell counting analysis for normal fat and WDLS samples.

In conclusion, we report the first use of OCT to characterize the tissue morphology of WDLS, DDLS, and normal fat in human tissues. We also demonstrate the capacity for OCT-based modality to differentiate between WDLS and normal fat samples based on numerical approach.

\section{References}

1. L. G. Kindblom, "Lipomatous tumors - how we have reached our present views, what controversies remain and why we still face diag- nostic problems-A tribute to Dr Franz Enzinger," Adv. Anat. Pathol. 13(6), 279-285 (2006).

2. P. C. W. Hogendoorn, F. Collin, S. Daugaard, A. P. Dei Tos, C. Fisher, U. Schneider, R. Sciot, and Eortc, "Changing concepts in the pathological basis of soft tissue and bone sarcoma treatment," Eur. J. Cancer 40(11), 1644-1654 (2004).

3. S. Singer, C. R. Antonescu, E. Riedel, and M. F. Brennan, "Histologic subtype and margin of resection predict pattern of recurrence and survival for retroperitoneal liposarcoma," Ann. Surg. 238(3), 358-370 (2003).

4. G. Lahat, J. E. Madewell, D. A. Anaya, W. Qiao, D. Tuvin, R. S. Benjamin, D. C. Lev, and R. E. Pollock, "Computed Tomography Scan-Driven Selection of Treatment for Retroperitoneal Liposarcoma Histologic Subtypes," Cancer 115(5), 1081-1090 (2009).

5. M. H. G. Katz, E. A. Choi, and R. E. Pollock, "Current concepts in multimodality therapy for retroperitoneal sarcoma," Expert Rev. Anticancer Ther. 7(2), 159-168 (2007).

6. W. Drexler and J. G. Fujimoto, Optical Coherence Tomography: Technology and Applications, SpringerLink, Berlin, New York, NY (2008).

7. J. S. Jelinek, M. J. Kransdorf, B. M. Shmookler, A. J. Aboulafia, and M. M. Malawer, "Liposarcoma of the extremities: MR and CT findings in the histologic subtypes," Radiology 186(2), 455-459 (1993).

8. M. J. Kransdorf, L. W. Bancroft, J. J. Peterson, M. D. Murphey, W. C. Foster, and H. T. Temple, "Imaging of Fatty Tumors: Distinction of Lipoma and Well-differentiated Liposarcoma," Radiology 224(1), 99-104 (2002).

9. S. Pilotti, G. D. Torre, A. Mezzelani, E. Tambroini, A. Azzarelli, G. Sozzi, and M. A. Pierotti, "The expression of MDM2/CDK4 gene product in the differential diagnosis of well differentiated liposarcoma and large deep-seated lipoma," Br. J. Cancer 82(7), 1271-1275 (2000). 